\title{
Variación morfológica poblacional de una especie invasora: el caracol gigante africano, Achatina fulica (Bowdich, 1822) (Mollusca: Gastropoda-Achatinidae) en el departamento del Valle del Cauca, Colombia
}

\author{
Morphological variation in the populations of an invasive species: the Giant \\ African Snail Achatina fulica (Bowdich, 1822) (Mollusca: Gastropoda-Achatinidae) \\ in the department of Valle del Cauca, Colombia
}

\section{Angie Patiño-Montoya, Oscar Murillo y Alan Giraldo}

\section{Resumen}

Las poblaciones de las especies invasoras pueden diferir en las características que determinan la intensidad de sus efectos sobre los ecosistemas nativos. El estudio de la variación morfológica aporta información valiosa sobre diferentes procesos evolutivos y ecológicos en las especies. Con el objetivo de evaluar los patrones de variación morfológica del caracol gigante africano (Achatina fulica), una especie invasora presente en el departamento del Valle del Cauca, se colectaron individuos en las zonas urbanas de 4 municipios (Buenaventura, Cali, Tuluá y Cartago). A estos especímenes se les realizó un análisis de morfometría geométrica y clasificación con base en el patrón de coloración. Se encontró que existe variación morfológica significativa dentro de la población, además del patrón de bandas o coloración, con un total de 13 patrones en toda la muestra. Se proponen las condiciones ambientales de cada localidad y los controles realizados por la autoridad ambiental como posibles causantes de la variación.

Palabras clave. Estructura etaria. Invasión biológica. Moluscos. Morfología. Patrón de coloración.

\begin{abstract}
Populations of invasive species may differ in the characteristics that determine the intensity of their effects on native ecosystems. The study of morphological variation provides valuable information on different evolutionary and ecological processes. In order to evaluate the patterns of morphological variation of the African Snail (Achatina fulica), an invasive species present in the department of Valle del Cauca, individuals were collected in urban areas of 4 municipalities (Buenaventura, Cali, Tuluá and Cartago). An analysis of geometric morphometry and classification based on the pattern of bands was performed on the collected specimens. A significant morphological variation within the population was found, along with a total of 13 patterns of bands or coloration in the entire sample. Environmental conditions of each locality and controls exerted by the environmental authority are proposed as possible causes of variation.
\end{abstract}

Keywords. Age range. Biological invasion. Color patterns. Morphology. Mollusks. 


\section{Introducción}

Las especies invasoras son consideradas como una de las principales amenazas para la conservación de la biodiversidad global debido a que ejercen presiones sobre la fauna local a diferentes niveles, lo que puede desencadenar procesos de extinción local, regional o global (Williamson, 1996; Mack et al., 2000). El estudio de la variación morfológica de estas poblaciones aporta información valiosa sobre procesos evolutivos y ecológicos que se desarrollan a nivel intraespecífico e interespecífico. Incluso, ha sido propuesto que las variaciones morfológicas en estas poblaciones son el resultado de la forma en cómo se relacionan los individuos que la conforman con el ambiente, siendo modulada la respuesta por la información genética de los individuos (Bookstein, 1996). En este sentido, Parker et al. (2003) han propuesto que las variaciones espaciales y temporales en las características morfológicas de las especies invasoras son generadas por plasticidad o adaptación local, por lo que se hace necesario que estas variaciones sean consideradas para entender y manejar esta presión biológica externa en los sistemas afectados.

El caracol gigante africano (Achatina fulica) es considerado una de las 100 plagas más peligrosas a nivel mundial por la Unión Internacional para la Conservación de la Naturaleza (UICN) debido a la alta proliferación, polifagia y simpatía por parte del ser humano (Patiño-Montoya y Giraldo, 2017). La invasión de esta especie puede generar consecuencias devastadoras en el medio ambiente, como lo es el desplazamiento de especies nativas, ya que este caracol es un competidor fuerte y disminuye cultivos o plantas nativas por la polifagia. Adicionalmente, esta especie puede afectar la salud pública al ser intermediario de varios parásitos, como los nemátodos del genero Angiostrongylus, que producen enfermedades letales como la meningitis eosinofílica y la angiostrongilyasis abdominal (Alburquerque et al., 2008, 2009; Liu et al., 2011).

A. fulica es una especie originaria de África oriental, que ha venido expandiendo su distribución desde hace aproximadamente 200 años (Fontanilla et al., 2014), y se ha establecido con éxito en todo el trópico y subtrópico del planeta (Alburquerque et al., 2008; Okon et al., 2012; De la Ossa-Lacayo et al., 2012; Avendaño y Linares, 2015). Como en la mayoría de moluscos, las diferencias en el tamaño, la forma y el patrón de bandas generalmente son atribuidas a respuestas adaptativas a nivel fisiológico y a la variación genética de la población, como una estrategia para afrontar las condiciones ambientales a las que se enfrentan (Okon et al., 2012). Particularmente, para el caracol gigante africano ha sido reportada una alta variación en la forma de la concha en lugares donde la invasión es antigua (mayor a 20 años), ya sea de tipo primario o secundario (reintroducción o desde un sitio introducido) (Pattarmanon, 2004; Sobrepeña y Demayo, 2014a). Sin embargo, aún no se ha evaluado la variación en la forma de la concha de esta especie en lugares donde la invasión es reciente (menor a 20 años) como en Ecuador, Perú, Argentina, Venezuela o Colombia (Alburquerque et al., 2008; Alburquerque et al., 2009; Goldyn et al., 2016; Goldyn et al. 2017). En este trabajo de investigación se presentan los resultados del análisis cualitativo y cuantitativo de la variación en la forma y patrón de coloración de la concha del caracol gigante africano, en una región de reciente invasión, el departamento del Valle del Cauca, suroccidente de Colombia.

\section{Materiales y métodos}

Área de estudio. La recolección de individuos se realizó en las ciudades de Buenaventura ( $\left.3^{\circ} 53^{\prime} \mathrm{N}-77^{\circ} 05^{\prime} \mathrm{O}\right)$, Cali $\left(3^{\circ} 26^{\prime} \mathrm{N}-76^{\circ} 31^{\prime} \mathrm{O}\right)$, Tuluá $\left(4^{\circ} 05^{\prime} \mathrm{N}-76^{\circ} 12^{\prime} \mathrm{O}\right)$ y Cartago $\left(4^{\circ} 44^{\prime} \mathrm{N}-75^{\circ} 54^{\prime \prime}\right)$ en el departamento del Valle del Cauca, Colombia. Buenaventura está situada en la región del Chocó Biogeográfico, a una altitud promedio sobre el nivel del mar de $7 \mathrm{~m}$. La vegetación dominante es de tipo de bosque muy lluvioso tropical, con registro de precipitación anual promedio de $7650 \mathrm{~mm}$, humedad relativa promedio del $89 \%$ y temperatura ambiental que varía entre $25^{\circ} \mathrm{C}$ 
y $28{ }^{\circ} \mathrm{C}$ (Rangel y Edgar, 2003). Las otras tres localidades se ubican en el valle geográfico del río Cauca, a una altitud promedio de 1000 m s.n.m. La vegetación dominante es de tipo de bosque seco tropical, con registros de precipitación anual promedio de $900 \mathrm{~mm}$, humedad relativa promedio del $70 \%$ y temperatura ambiental que varía entre $23{ }^{\circ} \mathrm{C}$ y $24{ }^{\circ} \mathrm{C}$ (Álvarez-López et al., 1984; Alberico et al., 2004) (Figura 1).

Métodos de campo. Los individuos utilizados para el desarrollo de esta investigación fueron recolectados en el marco de un estudio de dinámica poblacional de caracol gigante africano en el Valle del Cauca (Giraldo et al., 2014). En términos generales, el área urbana de cada una de las localidades fue dividida en celdas de $2 \mathrm{~km}^{2}$. Al

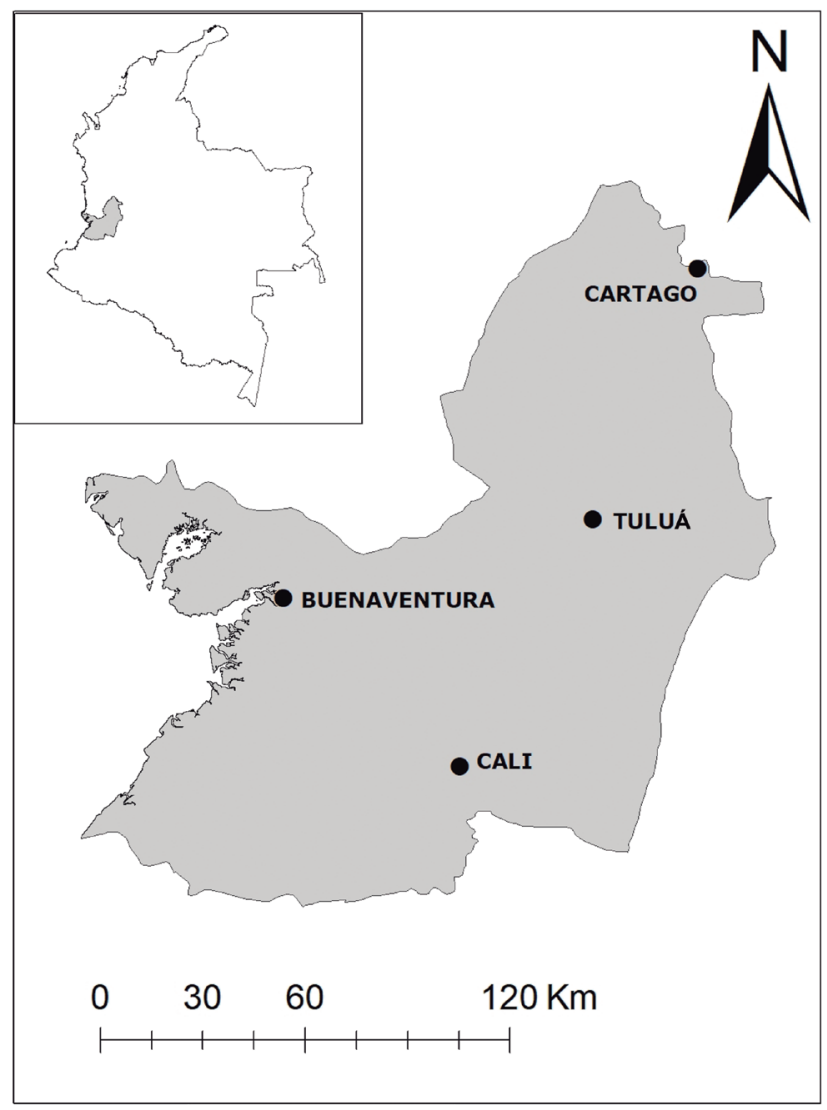

Figura 1. Localización geográfica de las localidades muestreadas en el departamento del Valle del Cauca (Colombia): Buenaventura, Cartago, Tuluá, Cali. interior de cada celda, se establecieron de manera aleatoria 3 parcelas de $16 \mathrm{~m}^{2}$, en donde se realizó el esfuerzo de búsqueda y recolección manual de caracoles vivos o conchas vacías, siguiendo las recomendaciones de seguridad para la manipulación de esta especie definidas por la autoridad ambiental.

Análisis morfométrico y de patrón de bandas. Con el propósito de establecer la estructura de talla de la población de A. fulica en la zona de estudio, se midió la altura total de la concha de cada individuo capturado y se construyó un histograma de talla considerando intervalos de $10 \mathrm{~mm}$. Se desarrolló un análisis de morfometría geométrica para evaluar la variación de la forma utilizando individuos adultos jóvenes con tallas entre 40 a 60 mm (Simão y Fischer, 2004), con el propósito de reducir la pérdida de información por desgaste de la concha y el efecto del crecimiento alométrico que presentan este tipo de especies.

Se realizó un registro fotográfico digital desde la vista ventral de 47 individuos: 12 de Buenaventura, 11 de Cali, 12 de Tuluá y 11 de Cartago y se utilizaron las herramientas de procesamiento digital para análisis morfométrico Thin-Plate Spline (TPS) (http://life.bio.sunysb.edu/ee/ rohlf/software.html). Se definieron 10 puntos de referencia distribuidos en el ápice, la sutura, la columela y la apertura de la concha (Figura 2). Para la transformación de las imágenes a formato TPS se utilizó TPSultil®. Posteriormente, se estableció la posición de los puntos de referencia para cada individuo utilizando TPSDig2 ${ }^{\circledR}$ y se procedió a realizar un análisis general de las deformaciones relativas utilizando la rutina TPSrelw ${ }^{\circledR}$. Este análisis se fundamenta en la comparación de la posición de los puntos de referencia, de todos los individuos, respecto a una forma consenso (Toro et al., 2010). Para determinar si la forma de las conchas es independiente del tamaño, se realizó un análisis de regresión con los dos primeros componentes resultantes del Análisis de Componentes Princi- 


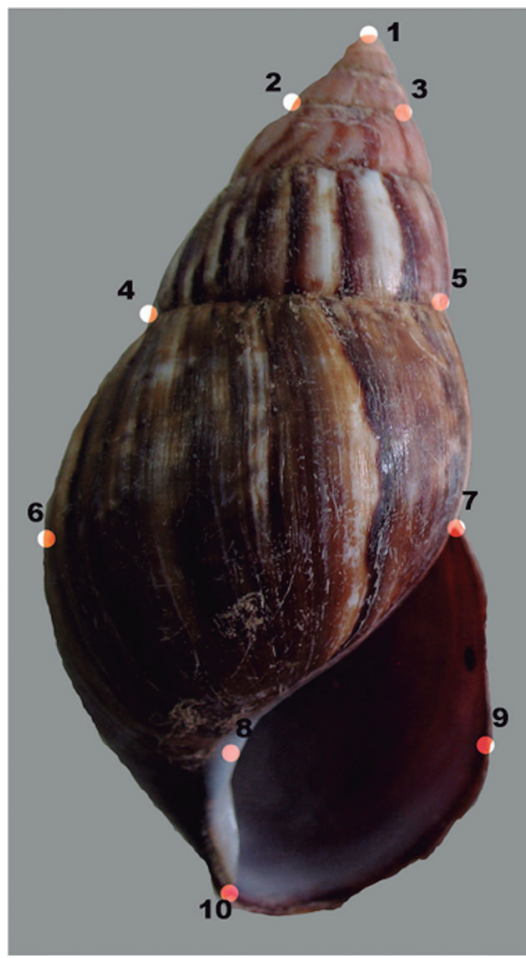

Figura 2. Landmarks digitalizados en orden numérico sobre las estructuras representativas de la concha de A. fulica: ápice, espiras, vuelta del cuerpo y apertura.

pales (ACP) de las deformaciones relativas como variables dependientes y el tamaño del centroide como variable independiente.

Para evaluar la variación entre localidades se realizó un análisis discriminante con base en las deformaciones relativas y posteriormente se compararon las localidades a través de un análisis de varianza (ANOVA) utilizando los valores de las dos primeras funciones discriminantes. Finalmente, se utilizó TPSsplin ${ }^{\circledR}$ para visualizar los cambios relativos de la forma de la concha que más contribuyeron a las funciones discriminantes establecidas en el análisis previo. Los análisis estadísticos se realizaron con los paquetes Vegan y MASS (Venables y Ripley, 2002).

Para el análisis del patrón de bandas se siguió la clasificación de Sobrepeña y Demayo (2014a), quienes definieron 14 patrones de bandas para la especie. Cada individuo fue clasificado de acuerdo con el patrón de bandas, describiéndose el patrón predominante para cada localidad y en toda la zona de estudio. Con el fin de evaluar si existe relación entre los patrones de bandas y las localidades, se realizó un análisis de contingencia y posteriormente pruebas chicuadrado secuenciadas en el programa PAST ${ }^{\circledR}$, para establecer cuales localidades eran la fuente de variación.

\section{Resultados}

Análisis morfométrico. Para realizar el análisis de variación de talla se procesaron un total de 192 conchas de A. fulica: 24 de Buenaventura, 39 de Cali, 80 de Cartago y 49 de Tuluá. Las poblaciones de Buenaventura y Tuluá presentaron los mayores promedios de altura total con $63,85 \pm 8,83 \mathrm{~mm} \mathrm{y}$ $63,25 \pm 12,97 \mathrm{~mm}$ respectivamente, seguidos de Cali con 53,71 $\pm 12,97 \mathrm{~mm}$ y Cartago con 39,18 $\pm 11,02 \mathrm{~mm}$. De acuerdo con los registros de tamaño, los adultos jóvenes de la zona de estudio no superaron el $60 \%$ del total de individuos capturados (Figura 3).

Los dos primeros componentes del ACP explicaron el $65 \%$ de la varianza contenida en las deformaciones relativas. La regresión entre estos

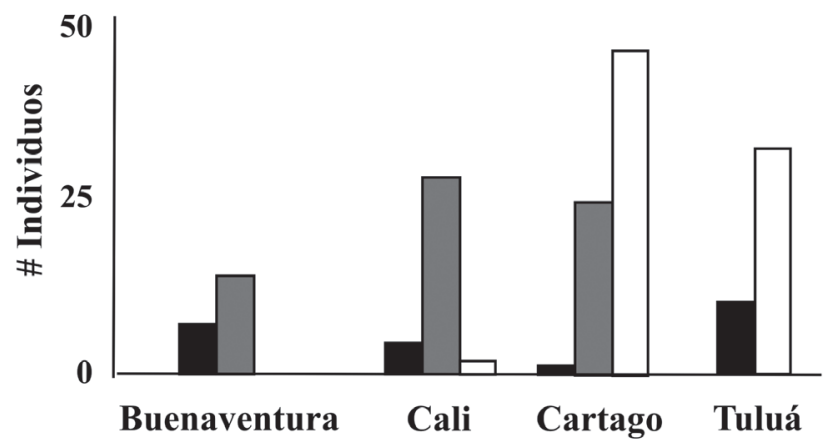

adulto $\square$ Adulto joven $\square$ Juvenil

Figura 3. Estructura etaria según Simao y Fisher (2004) de las conchas de Achatina fulica evaluadas en cada localidad. 
componentes y el tamaño del centroide no fue significativa $\left(\mathrm{F}_{(2,44)}=0,37, p=0,68\right)$, lo que indica que no existió una variación significativa en la forma de la concha en el rango de tamaño de los individuos estudiados (40-60 $\mathrm{mm})$, por lo que las variaciones en forma que sean detectadas serían independientes del tamaño.

El análisis discriminante indicó que el 70 \% de la varianza en la forma de la concha de $A$. fulica estuvo contenida en las dos primeras dimensiones. Las deformaciones relativas (RW) que más aportaron a la primera función discriminante (FD1) fueron el 2X y el 2Y, las cuales representaron el ancho de la apertura amplia, y el ancho y el tamaño de la espira. La segunda función (FD2) representó el tamaño de la espira y el ancho de la vuelta del cuerpo angosta (RW 4Y y 6Y). Por último, la tercera función (FD3) la orientación de la espira y del ápice (RW 1Y-2Y) (Figura 4, Tabla 1).

La primera función discriminante (FD1) proporcionó evidencia significativa de la diferencia de la forma de la concha entre las diferentes localidades de estudio $\left(\mathrm{F}_{(2,44)}=17,14, p\right.$
$<0,05)$, permitiendo identificar una diferencia de la forma entre los especímenes de Buenaventura con los especímenes de las localidades del valle geográfico del río Cauca (Figura 3). Entre estas últimas localidades la FD1 no sugirió la presencia de diferencias en la forma de la concha $(p=0,88$ y $p=0,08)$. Sin embargo, al incluir en el análisis la variación de la segunda $\left(\mathrm{FD} 2, \mathrm{~F}_{(2,44)}=8,08\right.$, $p 0,0001)$ y tercera $\left(\mathrm{FD} 3, \mathrm{~F}_{(2,44)}=8,08, p=0,0001\right)$ función discriminante, se detectaron diferencias significativas en la forma de la concha de A. fulica entre Tuluá y Cartago $(p<0,05)$ y Tuluá con Cali $(p<0,05)$.

En términos generales, la forma de la concha de los individuos de A. fulica que habitan en la ciudad de Buenaventura tiende a tener una apertura amplia y una espira delgada y alargada, lo que se traduce en una concha más estilizada con respecto a las poblaciones del valle geográfico del río Cauca. Por otra parte, la forma de la concha de las poblaciones de A. fulica que habitan en el valle geográfico del río Cauca tienden a variar entre sí en el ancho de la vuelta del cuerpo y en la orientación del ápice (Figura 5).

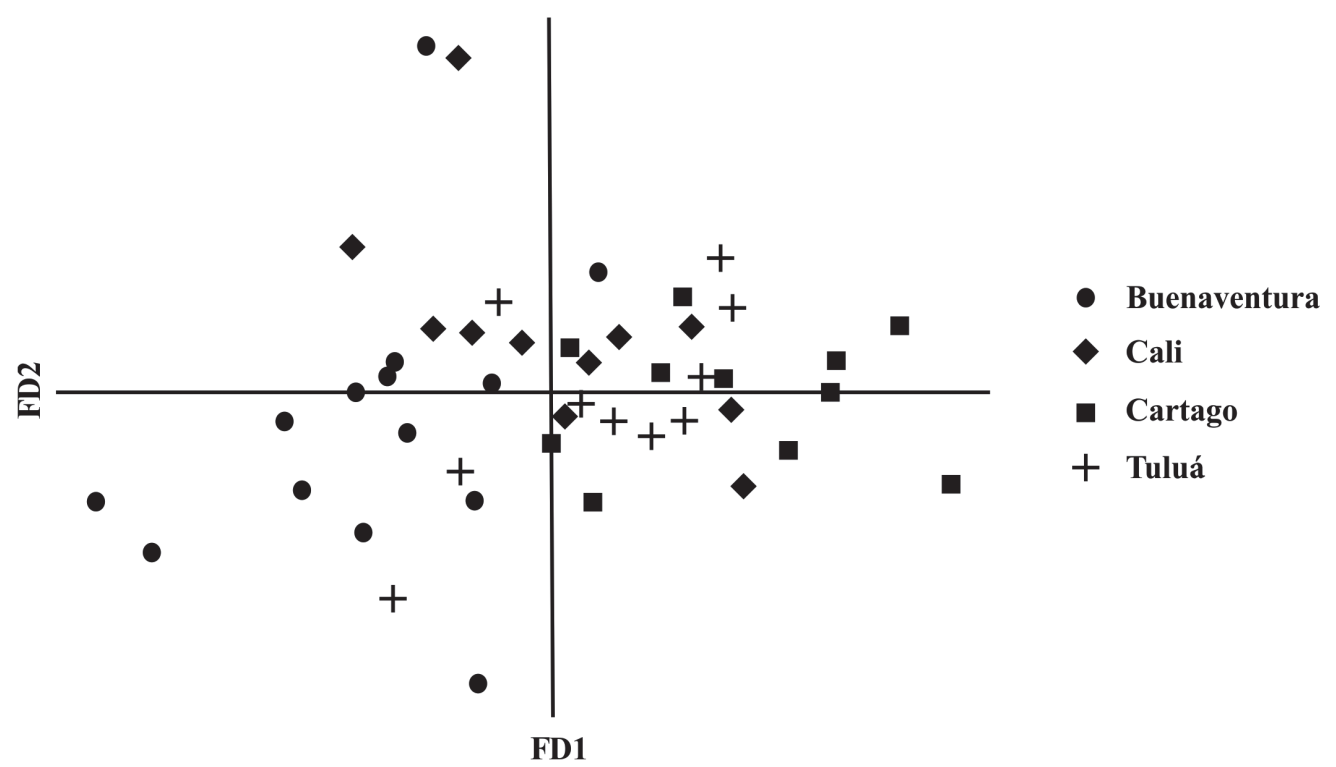

Figura 4. Análisis discriminante para las poblaciones de A. fulica muestreadas en cuatro municipios del Valle del Cauca. 
Tabla 1. Aportes de las deformaciones relativas a las funciones discriminantes FD1, FD2 Y FD3. LD1, LD2 Y LD3: Coefficients of Lineal Discriminants.

\begin{tabular}{cccc}
\hline & LD1 & LD2 & LD3 \\
\hline $1 X$ & $-8,35 \mathrm{E}-13$ & $6,97 \mathrm{E}-13$ & $-1,14 \mathrm{E}-12$ \\
\hline $1 \mathrm{Y}$ & $-4,20 \mathrm{E}-13$ & $-3,94 \mathrm{E}-13$ & $2,66 \mathrm{E}-12$ \\
\hline $2 \mathrm{X}$ & $1,02 \mathrm{E}-12$ & $-5,92 \mathrm{E}-13$ & $-1,04 \mathrm{E}-12$ \\
\hline $2 \mathrm{Y}$ & $-1,09 \mathrm{E}-12$ & $3,26 \mathrm{E}-13$ & $1,54 \mathrm{E}-12$ \\
\hline $3 \mathrm{X}$ & $-6,51 \mathrm{E}-13$ & $1,13 \mathrm{E}-13$ & $-3,68 \mathrm{E}-13$ \\
\hline $3 \mathrm{Y}$ & $1,80 \mathrm{E}-13$ & $8,14 \mathrm{E}-13$ & $5,44 \mathrm{E}-13$ \\
\hline $4 \mathrm{X}$ & $2,54 \mathrm{E}-13$ & $-1,53 \mathrm{E}-13$ & $3,59 \mathrm{E}-13$ \\
\hline $4 \mathrm{Y}$ & $-6,89 \mathrm{E}-13$ & $-2,68 \mathrm{E}-12$ & $1,33 \mathrm{E}-13$ \\
\hline $5 \mathrm{X}$ & $2,71 \mathrm{E}-13$ & $-5,68 \mathrm{E}-13$ & $-1,19 \mathrm{E}-12$ \\
\hline $5 \mathrm{Y}$ & $-4,74 \mathrm{E}-13$ & $2,01 \mathrm{E}-13$ & $-5,39 \mathrm{E}-13$ \\
\hline $6 \mathrm{X}$ & $7,55 \mathrm{E}-13$ & $1,40 \mathrm{E}-13$ & $2,78 \mathrm{E}-13$ \\
\hline $6 \mathrm{Y}$ & $4,83 \mathrm{E}-14$ & $1,75 \mathrm{E}-12$ & $-3,54 \mathrm{E}-13$ \\
\hline $7 \mathrm{X}$ & $-1,04 \mathrm{E}-12$ & $-5,31 \mathrm{E}-13$ & $7,94 \mathrm{E}-13$ \\
\hline $7 \mathrm{Y}$ & $-1,01 \mathrm{E}-13$ & $-1,96 \mathrm{E}-13$ & $5,23 \mathrm{E}-14$ \\
\hline
\end{tabular}

A.

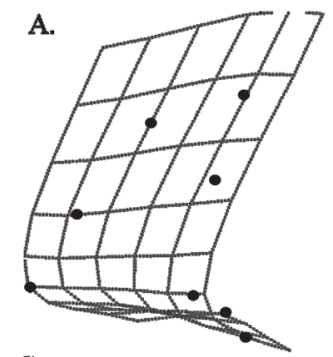

C.
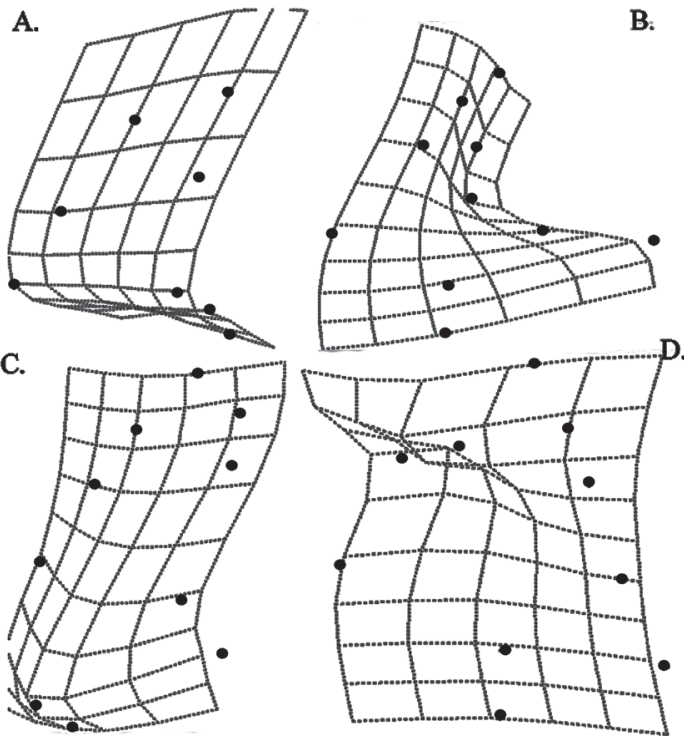

Figura 5. Formas de la concha de A. fulica asociadas a las funciones discriminantes FD1, FD2, FD3. A) PW1, B) PW2, C) PW4, D) PW6.
Patrón de coloración. De los 14 patrones de coloración que han sido descritos para el caracol gigante africano por Sobrepeña y Demayo (2014a), el único que no se encontró en la zona de estudio fue el patrón \# 14. Las localidades de Cali y Buenaventura obtuvieron la menor cantidad de patrones diferentes (6), a diferencia de Cartago y Tuluá en donde se registraron 11 y 10 patrones de bandas, respectivamente. El patrón \#5 fue el más abundante $(25,5 \%)$ en el área de estudio, seguido del patrón \#1 (22,9%). Sin embargo, este último patrón fue el más abundante para las localidades de Cartago y Tuluá (>30 \%), mientras que el patrón \#5 fue el más abundante en Cali $(33,3 \%)$ y \#7 (29,2 \%) y \#9 (20,8 \%) fueron los más abundantes en Buenaventura (Tabla 2, Figura 6). Es importante destacar que en ninguna de las localidades de estudio se registró el total de los patrones de bandas registradas en todo el Valle del Cauca (13 patrones).

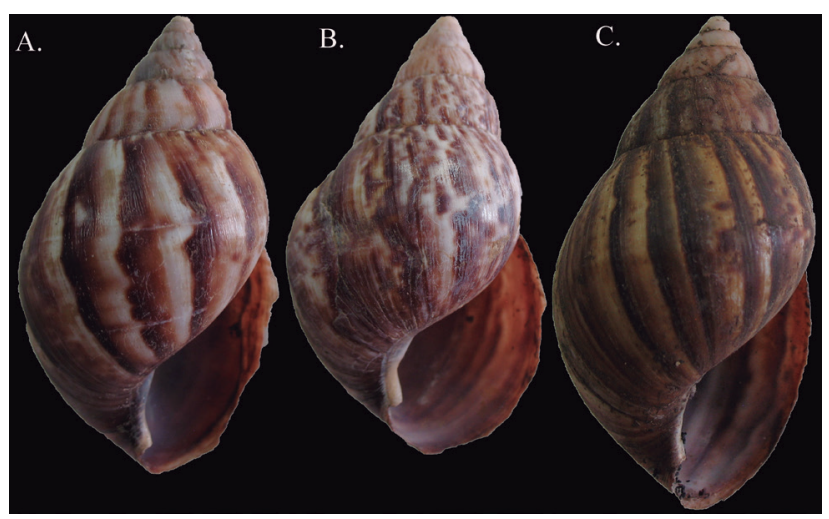

Figura 6. Patrones de coloración según la clasificación de Sobrepeña y Demayo (2014a) predominantes en los cuatro municipios del Valle del Cauca. A) Patrón 5 (Cali), B) Patrón 7 (Buenaventura) y C) Patrón 1 (Cartago y Tuluá). No se estableció independencia entre el patrón de coloración y las localidades $\left(\chi^{2}{ }_{(36)}=75,7, p 0,0001\right)$, siendo la frecuencias de los patrones de coloración significativamente diferentes entre localidades $\left(x_{(36)}^{2}=30, p\right.$ valor $\left.>>0,05\right)$. Este resultado sugiere que cada localidad tuvo un patrón específico de banda dominante y además cada localidad exhibió una frecuencia diferente de patrones de bandas presentes. 
Tabla 2. Frecuencia expresada en porcentaje de los patrones de bandas presentes en las conchas de Achatina fulica de las localidades de Buenaventura, Cali, Tuluá, Cartago y el total. n: número de conchas.

\begin{tabular}{lccccc}
\hline & Buenaventura & Cali & Cartago & Tuluá & Total \\
\hline Patrón 1 & 0,0 & 2,6 & 31,3 & 36,7 & 22,9 \\
\hline Patrón 2 & 4,2 & 0,0 & 3,8 & 0,0 & 2,1 \\
\hline Patrón 3 & 16,7 & 10,3 & 3,8 & 8,2 & 7,8 \\
\hline Patrón 4 & 0,0 & 0,0 & 2,5 & 6,1 & 2,6 \\
\hline Patrón 5 & 12,5 & 33,3 & 25,0 & 26,5 & 25,5 \\
\hline Patrón 6 & 0,0 & 0,0 & 0,0 & 2,0 & 0,5 \\
\hline Patrón 7 & 29,2 & 15,4 & 6,3 & 0,0 & 9,4 \\
\hline Patrón 8 & 16,7 & 12,8 & 11,3 & 2,0 & 9,9 \\
\hline Patrón 9 & 20,8 & 25,6 & 7,5 & 10,2 & 13,5 \\
\hline Patrón 10 & 0,0 & 0,0 & 2,5 & 4,1 & 2,1 \\
\hline Patrón 11 & 0,0 & 0,0 & 0,0 & 2,0 & 0,5 \\
\hline Patrón 12 & 0,0 & 0,0 & 5,0 & 2,0 & 2,6 \\
\hline Patrón 13 & 0,0 & 0,0 & 1,3 & 0,0 & 0,5 \\
\hline n & 24 & 39 & 80 & 49 & 192 \\
\hline
\end{tabular}

\section{Discusión}

Entender cómo reaccionan las especies invasoras, como el caracol gigante africano, a los factores bióticos y abióticos en un entorno foráneo es importante para establecer planes de control y manejo de este tipo de especies (Sakai et al., 2001; Davidson et al., 2011; Avendaño y Linares, 2015). Particularmente para los moluscos, las variables que más aportan información sobre las interacciones con el ambiente son las variaciones en tamaño y forma, de ahí la importancia de conocer los patrones de variación de estos atributos morfológicos en un área de interés (Mack et al., 2000; Davidson et al., 2011).

En este trabajo no se encontró evidencia en la fracción de tamaño estudiada de tendencia alométricas, por lo que es de esperar independencia entre la forma y el tamaño. Bajo este escenario, los patrones de variación en la forma y en coloración estarían siendo modulados por factores diferentes al tamaño. Factores como las condiciones climáticas, la estructura y distribución espacial de zonas verdes o lotes baldíos de las ciudades, múltiples eventos de introducción e incluso las medidas de control que realizan las autoridades ambiental locales y regionales, estarían afectando de manera significativa el tamaño y la forma de estos moluscos (Raut y Baker, 2002; Alburquerque et al., 2009; Avendaño y Linares, 2015). Además, las variaciones espaciales en la longitud de la concha encontradas en este estudio pueden deberse a las diferencias climá-ticas entre los ambientes ocupados por el caracol gigante africano en el Valle del Cauca, que va de un ambiente húmedo del Chocó Biogeográfico (Buenaventura) a un ambiente 
seco del valle geográfico del río Cauca (Cali, Tuluá, Cartago). Este resultado es similar al reportado por Giraldo et al. (2014) y Avendaño y Linares (2015), quienes proponen que las características ambientales presentes en la ciudad de Buenaventura tenderían a favorecer la tasa de crecimiento de esta especie, ya que no está sujeto a un fuerte estrés hídrico, lo que resultaría en conchas más grandes, con apertura amplia y espira delgada y alargada.

La disponibilidad de microhábitats que puede ocupar el caracol gigante africano en un ambiente urbano (lotes baldíos, jardines, fuentes hídricas o parques) puede ser un elemento que estaría promoviendo la variación del patrón de bandas al interior de cada localidad. De acuerdo con Giraldo et al. (2014), la disponibilidad de microhábitats favorables para el caracol gigante africano en las localidades de estudio es significativamente diferente, siendo mayor la disponibilidad de lotes baldíos en Buenaventura, mientras que en Cali, Tuluá y Cartago los jardines, separadores viales y zonas verdes asociadas a parques fueron más frecuentes. Como se ha reportado en otros moluscos, la complejidad estructural del ambiente donde se desenvuelvan está relacionado con la forma de crecimiento (Flores-Campaña et al., 2012). Por lo tanto, en el caso del caracol gigante africano, su relación con la disponibilidad de microhábitats en los centros urbanos con procesos de invasión reciente podría estar modulando el tamaño y el ancho de la espira, así como la vuelta del cuerpo (Tomiyama, 1992; Pattarmanon, 2004; Alburquerque et al., 2009; Sobrepeña y Demayo, 2014b).

Debido a la naturaleza invasora del caracol gigante africano, es probable que al departamento del Valle del Cauca estén ingresando individuos de diferentes lugares, en diferentes temporadas e incluso con diferente intensidad (Fontanilla et al., 2014; Patiño-Montoya y Giraldo, 2017). Lo anterior, sumado al control diferencial sobre clases específicas de tamaño de la población (adultos) como consecuencia de las acciones de control implementada por la autoridad ambiental, explicaría la constante movilización de los individuos dentro de la población que se encontró en el análisis discriminante. Finalmente, el patrón de coloración descrito en este trabajo para el caracol gigante africano en el Valle del Cauca podría ser el resultado de la interacción conjunta de forzantes genéticos y ambientales que estarían modulando las frecuencias de ocurrencia de patrones de bandas en cada localidad, e igualmente su variabilidad espacial (Sobrepeña y Demayo, 2014a, 2014b; Mack et al., 2000; Alburquerque et al., 2008; Pattarmanon, 2004; De la Rosa et al., 2010).

\section{Conclusión}

El tamaño del ápice, de la apertura y la forma de la vuelta de la concha del caracol gigante africano (Achatina fulica) en el Valle del Cauca fueron las fuentes de variación de la forma entre las localidades de estudio, además de que se establecieron diferencias espaciales significativas en los patrones de coloración de la concha. Considerando los resultados obtenidos, probablemente el efecto de esta especie invasora sobre los hábitats urbanos del Valle del Cauca no es uniforme. Por lo tanto, la capacidad de resiliencia de los sistemas urbanos afectados por la invasión del caracol gigante africano también sería variable.

\section{Agradecimientos}

A Rodrigo Lozano, Katherine Young, Viviana Collazos, Mónica Gómez y Natalia Rivera por su apoyo durante la realización de los muestreos. Este trabajo fue cofinanciado por la Corporación Autónoma Regional del Valle del Cauca (CVC) y la Universidad del Valle, a través del proyecto de investigación "Generación de Línea Base de Información sobre la Especie Invasora Achatina fulica en el Departamento del Valle del Cauca", Fase 2 Acuerdo 054 de 2014. A María Isabel Salazar (CVC) por sus recomendaciones durante el desarrollo del proyecto base. Angie PatiñoMontoya es estudiante del programa de maestría 
en Ciencias Biología de la Universidad del Valle y recibió asistencia de docencia como estímulo académico durante la investigación.

\section{Referencias}

Alberico, M., Saavedra, C. A. y García-Paredes, H. (2004). Criterios para el diseño e instalación de casas para murciélagos: Proyecto CPM (Cali, Valle del Cauca, Colombia). Actualidades Biológicas, 26, 5-11.

Alburquerque, F. S., Peso-Aguilar, M. C. y Assuncao-Alburquerque, M. J. T. (2008). Distribution, feeding behavior y control strategies of the exotic land snail Achatina fulica (Gastropoda: Pulmonata) in the northeast of Brazil. Brazilian Journal of Biology, 68(4), 837-842.

Alburquerque, F. S., Peso-Aguilar, M. C., Assuncao-Alburquerque, M. J. T. y Gálvez, L. (2009). Do climate variables and human density affect Achatina fulica (Bowditch) (Gastropoda: Pulmonata) Shell length, total weight and condition factor? Brazilian Journal of Biology, 69(3), 879-885.

Álvarez-Lopez H., Heredia, M. D. y Hernández, M. C. (1984). Reproducción del cucarachero común (Troglodytes aedon, Aves Troglodytidae) en el Valle del Cauca. Caldasia, 14(66), 85-124.

Avendaño, J. M. y Linares, E. (2015). Morfometría del caracol gigante africano Achatina fulica (Gastropoda: Achatinidae) en Colombia. Cuadernos de investigación, 7(2), 287-293.

Bookstein, F. (1996). Landmark methods for forms without landmarks: morphometrics of Group differences in outline shape. Medical Image Annals, 1(3), 225-243.

Davidson, A. M., Jennions, M. y Nicotra, A. B. (2011). Do invasive species show higher phenotypic plasticity than native species and, if so, is it adaptive? A meta-analysis. Ecology letters, 14(4), 419-431.

De la Ossa-Lacayo, A., De la Ossa, J. y Lasso, C. A. (2012). Registro del caracol africano gigante Achatina fulica (Bowdich 1822) (Mollusca:
Gastropoda-Achatinidae) en Sincelejo, Costa Caribe de Colombia. Biota colombiana, 13(2), 247252.

De la Rosa, V. G. D., Torres, M. A. J. y Demayo, C. G. (2010). Geometric morphometric tools in the analysis of shell shape of twelve local populations of the invasive snail Achatina fulica Bowdich from the Philippines Pp. 91-95. En Environmental Engineering and Applications (ICEEA), 2010 International Conference.

Flores-Campaña, L. M., Arzola-González, J. F. y de León-Herrera, R. (2012). Body size structure, biometric relationships and density of Chiton albolineatus (Mollusca: Polyplacophora) on the intertidal rocky zone of three islands of Mazatlan Bay, SE of the Gulf of California. Revista de Biología Marina y Oceanografía, 47(2), 203-211.

Fontanilla, I. K. C., Sta. María, I. M. P., García, J. R. M., Ghate, H., Naggs, F. y Wade, C. M. (2014). Restricted genetic variation in populations of Achatina (Lissachatina) fulica outside of east Africa and the Indian ocean islands points to the Indian ocean islands as the earliest known common source. PLoSONE, 9(9).

Giraldo, A., Bolívar, W. y González, A. (2014). Caracol africano en el Valle del Cauca: línea base para el Valle del Cauca (Informe técnico). Cali: Grupo de Investigación en Ecología Animal, Universidad del Valle. 30 pp.

Gołdyn, B., Guayasamín, P. R., Sanchez, K. A. y Hepting, L. (2016). Notes on the distribution and invasion potential of Achatina fulica Bowdich, 1822 (Gastropoda: Pulmonata: Achatinidae) in Ecuador. Folia Malacologica, 24(2), 85-90.

Gołdyn, B., Kaczmarek, L., Roszkowska, M., Guayasamín, P. R., Książkiewicz-Parulska, Z. y Cerda, H. (2017). Urban ecology of invasive giant african snail Achatina fulica (férussac) (gastropoda: achatinidae) on its first recorded sites in the Ecuadorian Amazon. American Malacological Bulletin, 35(1), 59-64.

Liu, C. Y., Song, H. Q., Zhang, R. L., Chen, M. X., Xu, M. J., Ai, L. y Lin, R. Q. (2011). Specific detection of Angiostrongylus cantonensis in the snail 
Achatina fulica using a loop-mediated isothermal amplification (LAMP) assay. Molecular and cellular probes, 25(4), 164-167.

Mack, R. N., Simberloff, D., Lonsdale, W. M., Evans, H., Clout, M. y Bazzaz, F. A. (2000). Biotic invasions: Causes, epidemiology, global consequences, and control. Ecological Applications, $10,689-710$

Okon, B., Ibom, L., Ettah, H. y Udoh, U. (2012). Comparative differentiation of morphometric traits and body weight prediction of Giant African Land Snails with four whorls in Niger Delta region of Nigeria. Journal of Agricultural Science, 4, 205-211.

Parker, I. M., Rodríguez, J. y Loik, M. E. (2003). An evolutionary approach to understanding the biology of invasions: Local adaptation and general-purpose genotypes in the weed Verbascum thapsus. Conservation Biology, 17, 59-72.

Patiño-Montoya, A. y Giraldo, A. (2017). Variación génica intrapoblacional del caracol gigante africano (Achatina fulica) en el Valle del Cauca. Revista MVZ Córdoba, 22(2), 5924-5936.

Pattamarnon, T. (2004). Shell morphological differences and genetic variation of the giant African snail Achatina fulica (BOWDICH, 1822) in Thailand (Tesis doctoral). Tailandia: Suranaree University of Technology. $250 \mathrm{pp}$.

Rangel, E. S. y Edgar, J. (2003). Analysis of Sea Level Data Sequences in Colombian Pacific and its Relationship to Climate Change. Meteorología Colombiana, 7, 53-66.

Raut, S. K. y Barker, G. M. (2002). Achatina fulica Bowdich and other Achatinidae as pest in tropical agricultura. En Barker, G. M. (Ed.). Mollusc as crop pest. Pp. 55-114. Hamilton, New Zealand: CABI publishing.
Sakai, A. K., Allendorf, F. W., Holt, J. S., Lodge, D. M., Molofsky, J., With, K. A. y McCauley, D. E. (2001). The population biology of invasive species. Annual review of ecology and systematics, 32(1), 305-332.

Simão, S. y Fischer, L. (2004). Estimativa e inferências do método de controle do molusco exótico Achatina fulica Bowdich 1822 (Stylommatophora; Achatinidae) em Pontal do Paraná, Litoral do Estado do Paraná. Cadernos da Biodiversidade, 4, 74-82.

Sobrepeña, J. M. y Demayo, C. G. (2014a). Banding pattern and shape morphology variations on shells of the invasive giant African land snail Achatina fulica (Bowdich 1822) from the Philippines. Annals of Biological Research, 5(1), 64-79.

Sobrepeña, J. M. y Demayo, C. G. (2014b). Outlinebased geometric morphometric analysis of Shell shapes in geographically isolated populations of Achatina fulica from the Philippines. Journal of Entomology and Zoology Studies, 2(4), 237-243.

Tomiyama, K. (1992). Homing behaviour of the giant African snail Achatina fulica (Ferussac) (Gastropoda; Pulmonata). Journal of Ethology, 10: 139-147.

Toro, M. V., Manriquez, G. y Suazo, I. (2010). Morfometría geométrica y el estudio de las formas biológicas: de la morfología descriptiva a la morfología cuantitativa. International Journal of Morphology, 28(4), 977-990.

Venables, W. N. y Ripley, B. D. (2002). Modern Applied Statistics with S. Fourth Edition. New York: Springer.

Williamson, M. (1996). Biological invasions. Vol 15. Reino Unido: Springer Science \& Business Media. 100 pp. 
Angie Patiño-Montoya

Universidad del Valle,

Facultad de Ciencias

angie.patino@correounivalle.edu.co

\section{Oscar Murillo}

Universidad del Valle,

Facultad de Ciencias

ecología.animal@correounivalle.edu.co

\section{Alan Giraldo}

Universidad del Valle,

Facultad de Ciencias

alan.giraldo@correounivalle.edu.co
Variación morfológica poblacional de una especie invasora: el caracol gigante africano, Achatina fulica (Bowdich, 1822) (Mollusca: Gastropoda-Achatinidae) en el departamento del Valle del Cauca, Colombia

Citación del artículo: Patiño-Montoya, A., Murillo, O. y Giraldo, A. (2018). Variación morfológica poblacional de una especie invasora: el caracol gigante africano, Achatina fulica (Bowdich, 1822) (Mollusca: GastropodaAchatinidae) en el departamento del Valle del Cauca, Colombia. Biota Colombiana, 19(1), 112-122. DOI: 10.21068/2018v19n01a07.

Recibido: 31 de agosto de 2017

Aprobado: 6 de marzo de 2018 